\title{
LA BASE DE LA METAFÍSICA DARWINANA
}

\section{DARWINIAN METAPHYSICS}

\author{
Carlos CAstrodeza* \\ Depto. Lógica y Filosofía de la Ciencia \\ Facultad de Filosofía \\ Universidad Complutense de Madrid
}

RESUMEN: Al contrario de lo que parece creerse en la actualidad, las consideraciones teóricas de Darwin sobre el origen de las especies estaban todo el tiempo de acuerdo con el pensamiento de sus colegas naturalistas. De hecho, para él era a todas luces imposible instrumentar sus propuestas radicales fuera del marco de la teología natural. Es más, aún el llamado crucial «efecto Malthus» se debe interpretar en el contexto de la evolución de la historia natural de la época. Finalmente, la aplicación de su teoría al origen del hombre tuvo peor destino que su a final de cuentas desesperados intentos de promoción de su teoría de la selección natural. El radicalismo de Darwin forma parte de la visión del mundo de la actualidad pero no era así en su tiempo. Es más, lo que se acepta hoy día es su pose cultural porque sus elucubraciones teóricas supusieron un callejón sin salida aunque, por supuesto, no así su excelente trabajo empírico como naturalista ajustado por entero a los cánones metaepistémicos de la época.

Palabras Clave: Evolución, teologia, Darwin, historia natural.

ABSTRACT: Contrary to seemingly current thought, Darwin's theoretical considerations on the origin of species were always in accord with the thought of his fellow naturalists.

* e-mail: castrode@filos.ucm.es 
In fact it was for all intents and purposes impossible for him to implement his radical presuppositions outside the framework provided by natural theology. Even the so called momentous «Malthus effect» has to be visualized in the context of the evolving natural history of his time and place. Finally, the theoretical application of his main idea to the origin of man was still more ill fated that his at all accounts desperate promotion of his theory of natural selection. Darwin's radicalism is part of today's vision on the world but was not so in his own intellectual surroundings. In fact, what is accepted today is his cultural standing because his theoretical speculations were a dead end by then, although his excellent empirical work as naturalist which deserves only praise fitted entirely the metaepistemic bill in his social context.

KEYWORDS: Evolution, theology, Darwin, natural history.

\section{La ubicación ontoepistémica de Charles Darwin}

\section{Generalidades}

Conviene puntualizar una vez más la tesitura darwiniana en el sentido que desde la celebración del primer centenario de su muerte en 1982, la hagiografización de su persona y obra es algo enteramente fuera de lugar por lo que procede remitirse una vez más a la historiografía pertinente del modo más ajustado posible tratando de eliminar todo presentismo (Whiggishness) al respecto y emoción admirativa excesiva hacia el ínclito inglés.

La creencia más generalizada entre naturalistas y pensadores en general en el entorno pre-darwinano, basada en una percepción afín de la realidad, es que el acoplamiento (adaptación) de los seres vivos a su medio es perfecto. La base de esa perfección radica en unas leyes naturales (propiciadas por un Creador a la sazón pero no así necesariamente ${ }^{1}$ ) para que todo permanezca en armonía natu-

1 Véase, como ejemplo significativo, la obra de los naturalistas escoceses Robert Edmond Grant o Robert Knox (Desmond, 1984, Secord, 1991), amén de las consideraciones del también escocés David Hume. Véase asimismo Gillespie (1979) y en contra de la interpretación exclusivamente teísta de Cornell (1987) 
ral, de manera que las variaciones del medio vienen acompañadas por variaciones en los seres vivos para que este acoplamiento se mantenga, es decir, más que una estructura rígida el conjunto «seres vivos-medio» se correspondería con una arquitectura flexible para amortiguar y en fin paliar las variaciones normales que acaecen debido a las estaciones y a las variaciones climáticas y geológicas en general. Además esta realidad sería sempiterna según, por ejemplo, Charles Lyell, el creador oficial de la geología moderna (Principles of Geology, 3vols., 1830-33, en su primera edición), o sea que dicha realidad siempre ha sido así y siempre lo será (uniformismo y actualismo) de acuerdo asimismo con una percepción afín de la realidad en cuestión, percepción no compartida en general en un principio por numerosos coetáneos ingleses del autor escocés (cuyas ideas se basaban asimismo en otro autor escocés anterior, James Hutton ${ }^{2}$ ) como puedan ser Adam Sedgwick de la Universidad de Cambridge o William Buckland de la de Oxford. Uno de los problemas es que tanto Sedgwick como Buckland, como sus numerosos seguidores, creían en una historia progresiva del mundo (todo va a mejor en la realidad circundante, no sólo en la humana). Para Lyell sin embargo la historia geológica sólo refleja constancia. Dichas ideas en su matiz más teológico (teología natural), muy acusado en la línea Sedgwick-Buckland, pero no tanto en Lyell, se remontan a la denominada revolución científica que ocurriera especialmente en el barroco británico y que vienen representadas por las especulaciones químicas de Lord Boyle, las físicas de Isaac Newton y las más propiamente pertinentes a la historia natural de John $\mathrm{Ray}^{3}$ (Crowther, 1982).

Darwin con modificaciones menores (incluidas sus ideas transformistas iniciales) participaba de esas creencias de su tiempo (especialmente de las de Lyell) que evolucionan a mayores hacia mediados de los años 50 del siglo XIX cuando Darwin ya se ha impregnado en buena medida del conocimiento al respecto y puede empezar a modificar el esquema ontoepistémico subyacente pero de acuerdo con nuevas interpretaciones que se basan en nuevas observaciones como es, por ejemplo, el reconocimiento del origen reciente del ser humano que el mismo Lyell acepta hacia 1859 sin admitir empero el origen propiamente animal en cuestión (Bynum, 1984). De hecho, ese origen animal no se reconoce en gene-

${ }^{2}$ Así como en las ideas geológicas de J. B. Lamarck (el asimismo fundador oficial del evolucionismo moderno).

3 Ray que introduce el término «especie» en la terminología científica además de suplir su obra maestra en teología natural (The Wisdom of God Manifested in the Works of Creation, 1691). 
ral siendo Darwin una de las excepciones en este sentido, pero excepción en su creencia más que en su demostración a pesar de su intento al respecto en The Descent of Man (1871) que resulta un tanto fallido para la ciencia de su tiempo en lo que desde una perspectiva histórica es una obra menor. La misma fenomenología transformista, huelga decirlo, se da profusamente en los coetáneos de Darwin (británicos y no británicos, Corsi, 2005) y uno de los problemas históricos que requieren explicación es la razón del protagonismo de Darwin en vida y especialmente después (Castrodeza, 2009, caps. I y II).

Darwin, como bien se sabe, tiene la preconcepción de que la variación de las especies no tiene un límite más allá del cual esa variación no es posible ${ }^{4}$ (lo que hoy día se denomina plateau en el contexto de la selección artificial), pero prácticamente tiene a toda la comunidad científica en su contra en principio, y digo en principio porque la percepción profunda de la comunidad científica es que la variación de las especies es limitada, aunque hay nuevas observaciones que apuntan a que esa variación puede efectivamente trascender los límites supuestos de manera que la comunidad científica va derivando en esa dirección y no únicamente Darwin. La diferencia es que para la comunidad científica en general se impone una cautela ontoepistémica, además de socialmente protocolaria, a la hora de interpretar ciertas observaciones que apuntan en una dirección transformista, cautela que también tiene Darwin aunque su interés específico en el tema (y un ansia desaforada de originalidad) le hace ser relativamente temerario al respecto. De manera que cuando Darwin publica la teoría transformista, en 1858-95, prácticamente toda la comunidad científico-teológica pertinente a la historia natural ${ }^{6}$ le sigue pero no porque realmente convenza a nadie, lo que efectivamente es el caso (su teoría transformista o no se acepta o se interpreta de un modo distinto a como la propone Darwin), sino porque muchos pensaban lo mismo más o menos conscientemente de manera que es como cuando en una bandada de pájaros uno emprende el vuelo y los demás le siguen. Y es que entre los naturalistas de la sociedad victoriana estaba muy mal visto especular teóricamente, había que ceñirse a datos observacionales y experimentales del modo más

${ }^{4}$ El origen posible de esa preconcepción se explícita en Castrodeza (1988, cap. II).

${ }^{5}$ En 1858 a nivel de artículo con Alfred Russel Wallace y en 1859 bajo la forma del librito que constituye su famoso Origin of Species.

${ }^{6}$ Entre los físicos (James Clerck Maxwell, Lord Kelvin) la situación es muy adversa a Darwin con la notable excepción de John Tyndall y aún así. 
ajustado y teóricamente aséptico posible y las publicaciones de las sociedades científicas más importantes como eran, además de la Royal Society, la Geological Society, la Linnean Society, la Zoological Society, la Geographical Society y la Entomological Society (por orden de importancia), entre otras de menor calado (como la más reciente Anthropological Society) normalmente no publicaban estudios eminentemente teóricos e ignoraban esas disquisiciones teóricas que no se pudieran fundamentar empíricamente de un modo directo como era el caso en torno a una teoría de la evolución de los seres vivos 7 . En la práctica las especies existían y no se podían modificar más allá de ciertos límites como se constataba indirectamente por selección artificial, y que en teoría esos límites se pudieran transgredir de aluna manera no era un tema que se ajustara al rigor científico del momento. Es más, las tres medallas que recibe Darwin en su entorno por la excelencia del trabajo realizado a la vuelta del famoso viaje del Beagle se corresponden a una medalla de la Royal Society concedida en 1853 por su trabajo en zoología (percebes vivientes y fósiles), otra (la medalla Wollaston) concedida en 1859 por la Geological Society por su trabajo en geología de años atrás y luego otra medalla de la Royal Society (la medalla Copley) concedida en 1864 por su trabajo en botánica (sobre los artificios por los cuales se fertilizan las orquídeas).

Por añadidura, la labor teórica que Darwin emprende entre los años 1837 y 1859 de un modo desigual (muy intenso en el trienio 1837-9, luego ya esporádicamente y otra vez de un modo bastante intenso en el quinquenio 1855-9 previo a la publicación de su libro) era principalmente la de interpretar el conocimiento biológico de su tiempo (morfología, embriología, paleontología, taxonomía, distribución geográfica, hibridación, instinto, etc.) en clave transformista lo que realmente no exigía grandes esfuerzos en el sentido de que la evidencia aun siendo muy abundante en general era tan escasa en áreas específicas que su reinterpretación a la luz del transformismo no requería en efecto un esfuerzo dialéctico especialmente intenso ${ }^{8}$ como se percibe en sus escritos al respecto y con especial claridad en el Origin of Species, sí había que hacerlo cuidadosamente, es decir, conociendo la ciencia de su tiempo, creando una propia

7 Véase Burkhardt (1974). De hecho en la ciencia anglo-americana siempre ha ocurrido así desde que John Locke, por ejemplo, abogara por la tolerancia para evitar conflictos violentos de orden teológico e incluso político, la fórmula es pues remitirse a los hechos (en este contexto véase Marshall, 2006).

8 Véase Ospovat (1981), p. 90. 
red de corresponsales incidentales por medio de una correspondencia continua más que amplia (la colección de sus cartas asciende a unas 14000) y todo ello a la luz del propio prestigio que Darwin supo ganarse con sus trabajos señalados como naturalista en ejercicio. Al mismo tiempo era importante hacer publicidad de la propia obra en los contextos culturales dominantes en Occidente, es decir, los contextos galo y alemán, además del inglés de ultramar (EEUU) de lo que Darwin también se preocupó muy mucho de promocionar. Sí, el «milagro» darwiniano se explica sin grandes aspavientos, aunque duela a sus numerosos admiradores (especialmente en nuestros días), y la darwinización del mundo con el tiempo no es más que el desarrollo de una teoría de la evolución naturalizada que se le endosa a Darwin como padrino extraordinario y como consecuencia de una reconstrucción histórica cuyo principal y posiblemente único precedente es la construcción del cristianismo a partir esta vez de unos datos históricos tan pobres como escasos. Darwinización que por su parte completaría la denominada mecanización del mundo a raíz de la tan traída y llevada revolución científica del renacimiento y barroco. Pero en el caso de Darwin los datos históricos por el contrario sobran, por así decirlo, lo que proclama que la reconstrucción histórica no depende tanto de la base fáctica como de lo que se quiera promover psicosocialmente? .

Como iban haciendo sus coetáneos, Darwin concluyó en los años 50 más por razones transformistas que por razones estructuralistas que la adaptación o acoplamiento con el medio nunca llegaba a ser perfecta/o. Se desfavorecía así la noción de armonía/equilibrio en la naturaleza y a cambio se potenciaba la idea de progreso y de que las cosas iban naturalmente a mejor (con temidos altibajos), algo en lo que participaba prácticamente toda la comunidad británica pero siguiendo no el liderazgo epistémico en clave positivo-optimista de Darwin sino el del filósofo Herbert Spencer (para la influencia colosal de este creador de un evolucionismo psicosocial de salón véase Francis, 2007) que a su vez se basaba en la obra embriológica del alemán Ernst von Baer así como del francés Henri Milne Edwards autores clave asimismo, como no podía ser de otra manera, en el evolucionismo darwiniano.

9 Un botón de muestra al respecto tan reciente como interesante es la publicación del Cambridge Companion to the "Origin of Species» (Ruse y Richards, eds., 2009) en que de 18 contribuciones de expertos sólo hay una que no es especialmente laudatoria, la del gran historiador de la biología Robert Olby aunque concluya su artículo con un tradional «eppur si muove» por si acaso. 


\section{La historia natural en la que Darwin se ve inmerso}

Para empezar, el mundo de la historia natural en la primera parte del siglo XIX centroeuropeo (Gran Bretaña, Francia y el entorno germanoparlante) es mayoritariamente fijista con una sujeción a una diversificación tipológica, es decir, las especies son estructuras fijas, o tipos, que varían como se ha dicho dentro de un margen más o menos amplio pero limitado. La aparición de nuevas especies es un fenómeno misterioso sujeto a esquemas creacionistas no necesariamente teológicos ${ }^{10}$, es decir, existe un creacionismo secularizado que se explicita como tal en el mundo angloparlante sin entrar en detalles que no se han observado de momento (Lyell), no se explicita en cambio en la realidad francoparlante (para Cuvier la cuestión del origen simplemente no es científica) y se mantiene confuso en el entorno germanoparlante dentro del contexto de la Naturphilosophie ${ }^{11}$. Simultáneamente, el determinismo ambiental de por ejemplo un Cuvier o un Lyell va cediendo el paso conceptual a una teleología de segundo orden por la que las estructuras vivientes no parecen corresponderse con precisión alguna con los medios que las acogen, es decir que siguiendo directrices alemanas no es la función la que delimita la forma sino al revés (múltiples y diferentes formas pueden tener funciones análogas).

Darwin después de aproximadamente dos años de especulaciones transformistas (aproximadamente desde algo antes julio de 1837 — red notebook — en que inicia sus cuadernos de notas A — geológico- y B - propiamente biológico- hasta finales de 1838-principios de 1839 en que da con una «teoría con la que poder trabajar») creyó resolver el problema del origen de las especies por

${ }^{10}$ Gillispie (op.cit.). Además hay que tener en cuenta que en la esfera específicamente teológica existía un creacionismo a voluntad o creacionismo especial por el que las especies se creaban «sobre la marcha» como si dijéramos y un creacionismo a partir de segundas causas o leyes naturales en el que la creación seguía pautas como cualquier otro acontecimiento físico, este creacionismo se confundía con el secularizado en sus efectos.

${ }^{11}$ De hecho Lorenz Oken publica su Lehrbuch der Naturphilosophie el mismo año, 1809, que Lamarck publica su Philosophie Zoologique. Si hay que elegir un trío de biólogos transformistas en el siglo XIX, Lamarck y Darwin estarían en un plano realista y el no menos importante Oken lo estaría en el plano idealista cuya traducción al plano realista le convierte en una influencia más que notable en el pensamiento evolucionista sobre toda en la fase formalista, opuesta a la funcionalista, lo que tuvo realmente una influencia decisiva en la construcción del pensamiento evolucionista. 
medio de su principio de selección natural que explicaba no sólo su evolución sino su adaptación. Para los coetáneos de Darwin la adaptación empero no era un problema a explicar sino simplemente una explicación en si misma que define a los organismos, o sea que o bien Dios crea a los organismos de manera que puedan sobrevivir o bien simplemente es una cuestión que ni se plantea directamente en esos términos teológicos, es decir, los organismos son estructuras equipadas para sobrevivir, equipamiento que se propicia por leyes naturales como las que en su día propuso el conde de Buffon en Francia en un marco naturalista (de manera que un medio idóneo propiciaría la formación de estructuras complejas en efecto idóneamente estables lo que equivaldría a su supervivencia provisional). La selección natural se contemplaba tácitamente por todos en el sentido que los organismos se acoplaban, como ya se ha indicado, dentro de un margen de variación más o menos amplio a las variaciones del medio. Prácticamente nadie intuía ${ }^{12}$ — salvo Darwin un tiempo después de su relectura de la obra principal del Rvdo Thomas Robert Malthus (An Essay on the Principle of Population, publicado anónimamente en su primera edición en 1798) a finales de septiembre de 1838 — la realidad de un proceso de selección natural indefinido porque nadie podía figurarse que un organismo se «deformara» indefinidamente para paliar las variaciones del medio. Esto último iba contra toda lógica fisiológica, por eso Darwin en su momento insiste constantemente sobre que las variaciones favorables que se pueden ir acumulando por selección natural siempre son minúsculas, como si esa pequeñez le facilitara al organismo su propio ajuste fisiológico-anatómico gradualmente muy poco a poco o sea como si un ajuste a cámara lenta fuera más creíble para Darwin (específicamente) que otro a grandes trazos ${ }^{13}$.

Dentro de esas ideas generales habría que añadir que la disyuntiva, según el registro geológico, de que los peces, los reptiles y los mamíferos hayan aparecido sobre la tierra en este orden es, según criterios creacionistas generales de la época, a causa de que esas respectivas clases de seres vivos se acomodaban per-

12 Digo prácticamente porque hubo un naturalista Patrick Matthew que publicara en 1831 un libro sobre madera para construir barcos e incidentalmente proponía una idea de selección natural indefinida a la que ni él ni nadie dieron la menor importancia aunque una vez que Darwin consiguió la fama Matthew hizo valer su originalidad, bastante en vano por cierto.

13 De hecho nadie entendía la insistencia de Darwin al respecto (ni siquiera su íntimo amigo, el joven e influyente científico Thomas Henry Huxley que se decantaba por el saltacionismo o «macromutacionismo», término de introducción muy posterior). 
fectamente a las condiciones ambientales del momento, condiciones que también harían su aparición en ese orden. En las palabras del gran teórico de la ciencia del momento, el Newton de biología, Georges Cuvier (Darwin, puestos a emplear esa iconografía procedente de la física, sería en todo caso el Einstein, valga el anacronismo), las "condiciones de existencia» propiciaban enteramente la estructura funcional del ser vivo (Cuvier, Le Règne Animal, 1817).

Como alternativa al pensamiento funcional (teleológico) de Cuvier, la existencia en el muy influyente contexto galo de la alternativa propiamente estructuralista, de acuerdo con la Naturphilosophie alemana asumida por Étienne Geoffroy Saint Hilaire, también tenía su importancia. Geoffroy proponía que todos los animales estaban compuestos esencialmente de los mismos elementos estructurales y que las estructuras orgánicas concretas de un animal se explicaban principalmente no por sus funciones sino más bien en referencia a las estructuras de otros animales. De hecho, el objetivo de la anatomía comparada era descubrir las «semejanzas filosóficas» entre los organismos (Philosophie Anatomique, 2 vols., 1818-22).

Las ideas de Cuvier eran en primera instancia enormemente afines a las de los naturalistas británicos que respiraban en la atmósfera naturalista de la teología natural. Pero a pesar del triunfo de Cuvier en el famoso debate que éste tuvo con Geoffroy en la Academia de Ciencias de París en 1830, las ideas de éste último se iban imponiendo en suelo británico debido en buena parte a la influencia de la embriología alemana porque mediante la misma se cotejaba que las formas de los distintos organismos a nivel embriológico tenían un parecido mucho más estrecho que en las formas adultas. Pero claro las formas adultas procedían del desarrollo de las embriológicas de tal manera que el dominio de la forma sobre la función desde la perspectiva germana tampoco se podía pasar por alto por mucho que la adaptación de los seres vivos a su medio se antojara que fuera tan perfecta que era la funcionalidad orgánica la que predominaba sobre la «formateadora» hasta quizá llegar a anularla por completo.

\section{Evolución de la dicotomía función-forma}

Los antecedentes metafísicos del funcionalismo tanto de Cuvier como de Lyell así como de la mayor parte de la teología natural de la primera mitad del siglo 
XIX se centran en la idea cristiana de que la obra del Creador es maleable hasta el extremo de que las mismas leyes naturales son contingentes ${ }^{14}$. Por su parte el formalismo germano tipificado en la obra de Lorenz Oken (Lehrbuch der Naturphilosophie, 1809) tiene sus raíces últimas en el estoicismo panteísta grecorromano que alcanzaría una versión épica en la ética de Spinoza y desembocaría finalmente en la Naturphilosophie germana, de tal modo que todo lo que somos y lo que nos rodea sería un reflejo arquetipal del arquetipo de los arquetipos que sería el principio divino que lo invade todo y lo formatea a su imagen y semejanza (Wolfgang Goethe). La secularización del mundo, o su desencanto en la terminología de Weber, tiene como antecedente más natural el panteísmo más pagano que judaico-cristiano de los herméticos alemanes opuesto al personalismo cristiano de la teología natural, y en este sentido no es que la influencia alemana se deje sentir cada vez más en la interpretación del mundo de la vida en el entorno darwiniano, sino que es la revolución burguesa con su rechazo inicial y progresivo de la autoridad de la iglesia de Roma y su enraizamiento en una actitud protestante la que endiosa al hombre occidental progresivamente y en esa tesitura seculariza su metafísica. La consecuencia no es que la realidad se perciba (o se cree) de distinta manera de un modo paradigmático kuhniano por ejemplo, sino que más bien son ciertos aspectos de la realidad los que se resaltan sobre otros ${ }^{15}$.

De manera que los formalistas no percibían en la adaptación orgánica (cuyo ajuste se suponía perfecto en un principio) una relación estrictamente funcional entre la forma orgánica y las condiciones inorgánicas. El famoso especialista en anatomía comparada inglés Richard Owen — que se hiciera cargo de la parte paleontológica de la zoología del Beagle por decisión de Darwin- fue uno de los pioneros ingleses a la hora de adoptar esa teleología de segundo orden (ver especialmente On the Nature of Limbs, 1849) que posibilitaba enormemente una interpretación transformista de la evolución de la vida, porque si todo deriva de una forma (arquetipo) inicial y las formas están todas ellas íntimamente relacio-

${ }^{14}$ Ideas trazables a los franciscanos oxonianos de los siglo XIII-IV (notablemente Roger Bacon y William Ockham). Por otra parte el rigor científico dentro del marco de la Teología Natural no es más metafísico que en otros marcos más secularizados e incluso que el marco en el que opera Darwin (Blaisdell, 1982, ver también Stenhouse, 1990).

15 Para una versión actual de la dicotomía forma-función puede consultarse Laubichler $e t$ al. (2008) así como Saladin (2009) y por supuesto el clásico de Russell (1916) y el mucho más actual pero no menos clásico de Gould (1977). 
nadas la derivación real de las unas a partir de las otras no requiere disquisiciones epistémicas profundas ${ }^{16}$. Es más, Owen introduce oficialmente los conceptos de analogía y homología orgánicas lo que facilita aún más una posible derivación transmutacionista. El mismo Owen se convierte en un cripto-transformista cuyo problema en este sentido es el de todos, ¿por qué mecanismo se puede llevar a cabo la posible derivación orgánica? La solución general que daría Darwin no convence a nadie y el mismo Darwin no contrarresta las críticas ni a satisfacción siquiera de sus seguidores más «leales» (Joseph Dalton Hooker, Thomas Henry Huxley, Charles Lyell, Asa Gray, Ernst Haeckel, John Lubbock, Alfred Newton, Francis Galton o Alfred Russel Wallace). Pero la concepción formalista de Owen ya se estaba mascando en suelo inglés en el mismísimo seno de la teología natural como se tipifica en el tratado Bridgewater de Peter Mark Roget, a los efectos de demostrar la gloria de Dios en su obra creadora (Animal and Vegetable Physiology, 1834). En este sentido el artículo que publicara el teólogo-naturalista Martin Barry dos años después o sea en 1836 — justo cuando volvía Darwin del viaje del Beagle("On the unity of structure in the animal kingdom», Edinburgh New Philosophical Journal, 22, 1836-7: 116-41, 345-64) dejaba el tema más que claro subrayando que para conocer la naturaleza en profundidad hay que centrarse en el estudio de la forma unitaria subyacente dejando a un lado la diversidad de las características adaptativas. Las ideas de Barry las incorpora el experto en fisiología comparada inglés William Benjamin Carpenter en una de sus obras capitales (Principles of General and Comparative Physiology, 1839) ${ }^{17}$. Del mismo modo el zoólogo de Harvard, el suizo Louis Agassiz, educado en Alemania y creacionista hasta la muerte expande las ideas de Carpenter y Owen en su magnífico An Essay of Classification de 1859 (el mismo año que Darwin publica su Origin). Se ve que aunque las ideas "paganas» provenientes de los países germanoparlantes propicien una secularización del mundo la compatibilización con la teología vigente en sus diferentes variantes no está de ningún modo descartada.

16 En realidad tanto Owen, como von Baer o Milne Edwards se situaban ontoepistémicamente en una situación intermedia entre Cuvier y Geoffroy en el sentido que como Cuvier admitían cuatro estructuras animales básicas (embranchements: vertebrata, articulata, mollusca y radiata) pero no admitían ni una explicación estrictamente teleológica de las mismas ni tampoco admitían que esos euatro diseños básicos tuvieran un referente común todavía más básico como pensaba Geoffroy.

17 Incidentalmente tanto Carpenter, como el botánico americano de Harvard, Asa Gray (íntimo amigo de Darwin) y, que se sepa, la única persona que Darwin realmente odió, el importante biólogo católico inglés Saint George Mivart, pasaron a ser los defensores más acérrimos en suelo inglés de una concepción teológica de la teoría de la selección natural. 
Darwin a su vuelta del viaje del Beagle más que dedicarse a especular sobre como aparecen, se mantienen y se extinguen las especies lo que hace es estudiar la situación y va leyendo entre otros escritos las obras mayores que se han ido mencionando. Su punto de referencia es la transmutación de las especies, idea que se adquiere más que pensando sobre el tema, como se suele especular, se fomenta en vez con motivo de un contencioso personal con base teológica que mantiene con el capitán del Beagle, Robert FitzRoy, ya que lo que éste cree que son especies en las distintas Islas Galápagos, Darwin piensa que son variedades, y al darle la razón los expertos (especialmente el ornitólogo John Gould) a la vuelta a Inglaterra a FitzRoy, la única salida airosa para Darwin es contemplar un teoría transmutacionista aparte de que el incidente a todas luces le crea un trauma teológico ${ }^{18}$. En fin, el caso es que pensar sobre las especies desde una perspectiva tanto fijista como transmutacionista tiene en la época problemas claros. Darwin antes de volver a Inglaterra es funcionalista en la línea de LyellCuvier, luego a su llegada se va pasando como casi todos los naturalistas de su entorno a la línea Carpenter-Owen-Agassiz (que es también la de von Baer-Milne Edwards).

Una de las dificultades que más le acosan a Darwin es también un problema para la mayoría de los naturalistas de la época y es que la adaptación de los organismos no se corresponde con su distribución geográfica, esta circunstancia es especialmente onerosa para la línea Lyell-Cuvier pero también lo es para la tendencia Carpenter-Owen-Agassiz y por supuesto para la línea transmutacionista a la que Darwin se aplica especialmente. En general en la línea Cuvier-Lyell predomina fundamentalmente un principio adaptativo (una ley de adaptación) mientras que en la otra línea ya se inmiscuye con fuerza un principio de herencia (una polarizing force en la terminología de Owen), es decir, por las reglas ya más explícitas de homología-analogía que proclama Owen los organismos están formalmente emparentados, pero claro Darwin piensa que el «formalmente» equivale a un «realmente» pero ¿cómo ocurre? Las contestaciones que esgrimen los evolucionistas más destacados de los tiempos de Darwin no convencen, fundamentalmente se trata del alemán Christian Leopold von Buch (el aislamiento potencia con el tiempo la esterilidad entre las poblaciones aisladas que conse-

18 Véase de nuevo Castrodeza (op. cit.). Curiosamente este incidente que es el que le coloca a Darwin en una pendiente clara hacia el libre pensamiento ni siquiera se menciona de pasada en la copiosísima literatura dedicada al tema (véase por ejemplo Brown, 1986). 
cuentemente se transforman en especies, véase por ejemplo su Physicalische Beschreibung der Canarischen Inseln, 1825) y, por supuesto, se trata asimismo del francés Jean Baptiste Chevalier de Lamarck (Philosophie Zoologique, 1809) según cuyas ideas un besoin intérieur a la manera de una ley natural físico-química potenciaría la evolución de las especies ${ }^{19}$. Una manera novedosa de contestar al «¿cómo ocurre?» aparece en una obra de carácter evolucionista que publica anónimamente un librero escocés, Robert Chambers, en 1844, Vestiges of the Natural History of Creation (año en que Darwin completa un ensayo de unas 259 páginas sobre su teoría después de siete años largos de especulaciones sobre el asunto). Chambers, basándose especialmente en el pensamiento embriológico más respetado en la época, el del Naturphilosph ya mencionado Karl Ernst von Baer (véase especialmente su Ueber Entwickelungsgeschichte der Thiere, 2 vols., 182837) propone que por leyes naturales concretas en ciertos organismos y en ciertas circunstancias el período de gestación se prolonga dando lugar a nuevas formas (véase la edición introducida por Secord, 1994).

El problema con la obra de Chambers es por un lado su anonimidad epistémicamente "culpable» y por otro que su obra contiene consideraciones que en parte no están de ningún modo al día ni en clara consonancia con el pensamiento oficial de la ciencia del momento. De cualquier modo se sabe hoy que a pesar de las críticas feroces que recibe la obra de Chambers por parte de los prebostes científicos del momento su éxito a nivel general (popular) es notable. No sólo alcanza su obra en vida por ejemplo el doble de ediciones que la obra fundamental que Darwin publicara en 1859 sino que científicos importantes como el mencionado Carpenter además de Alfred Russel Wallace (el oficialmente co-descubridor con Darwin de la teoría de la selección natural) se sienten en líneas generales convencidos por Chambers en lo que concierne a una evolución de los seres vivos. Más adelante y anteriormente a Darwin el filósofo inglés Herbert Spencer se siente también convencido por las ideas de Lamarck al leer la crítica de las mismas que hace Lyell en el segundo volumen de sus Principles.

19 Desde luego cuando Darwin propone su teoría de la selección natural y las críticas son cada vez más negativas a medida que van apareciendo las distintas y escasas ediciones de su Origin, Darwin va diluyendo el papel de la selección natural y dando cada vez más importancia al aislamiento y sobre todo a la idea de Lamarck que ese besoin interieur induce la adición de determinados comportamientos que se transforman en hábitos heredables y que propician cambios pertinentes estructurales en la anatomía y fisiología de los seres vivos. 
O sea que se ve que la idea transmutacionista se maneja y se cree en ella entre bastidores (como ya se ha dicho, Owen también se inclina aunque muy en privado por esas ideas de momento heterodoxas, en cambio el rechazo de Agassiz hacia las mismas es total y absoluto). El caso es que lo que no suscribe nadie son los mecanismos aducidos por Chambers. Se piensa cada vez más por la mayor parte de teólogos naturales y naturalistas en general que de alguna manera la evolución tiene que ocurrir pero no se sabe cómo (es el «misterio de los misterios» alega el conocido científico y astrónomo John Herschel), la propuesta de Darwin-Wallace de la selección natural no convence tampoco a nadie (aunque sus proponentes, especialmente Darwin, tengan credenciales científicas impecables), y aquéllos que se sienten convencidos por Darwin como el botánico de Harvard ya mencionado Asa Gray, o los conocidos teólogos naturales ingleses Baden Powell o Charles Kingsley, amén de otros muchos, le dan al mecanismo de DarwinWallace una interpretación estrictamente teológica (Dios iría actualizando su creación por un proceso de selección natural). El mismo Wallace se decanta por la teología en lo que concierne al origen del hombre y hasta Karl Marx que aunque en ningún momento acuda a la teología sí piensa con el tiempo que la tesis de Darwin se fundamenta en una ideología capitalista y no vale para el hombre. El pensador alemán acude al respecto a las ideas menos radicales, aunque más aventuradas, del pintor, fotógrafo, etnólogo y arqueólogo evolucionista galo Pierre Trémaux ${ }^{20}$.

\section{La saga personal de Charles Darwin}

\section{Antes de releer a Malthus}

La línea Carpenter-Owen-Agassiz le condujo a Darwin a Geoffroy. Es más Darwin como la mayor parte de los naturalistas ingleses, sobre todo los más jóvenes, simpatizaban por las ideas de Geoffroy en su debate perdido con Cuvier. Además, Geoffroy tenía una inclinación clara hacia el transmutacionismo lo que a los ojos de Darwin le reivindicaba todavía más. Una anomalía de difícil resolución para la línea Cuvier-Lyell venía constituida por la existencia de órganos rudimentarios porque su cometido funcional no se puede dilucidar con facili-

${ }^{20}$ Para un interesante trabajo al respecto véase Beck (2009), pp. 306-13. 
dad alguna, sin embargo su significado formalista era fácilmente asimilable a la línea Carpenter-Owen-Agassiz y por implicación a la línea que se puede ya denominar como Chambers-Darwin-Wallace, porque de hecho Darwin no estaba sólo en sus especulaciones aunque por edad, formación y experiencia personal fuera el centro de gravedad de la tesis transformista en su versión más naturalizada. La diferencia esencial entre la línea formalista y la propiamente transmutacionista es que en la primera existía mucha más cautela científica que en la segunda además de una cierta mística procedente del panteísmo metafísico de su idealismo inicial. En efecto, la línea funcionalista tenía muchas más dificultades tanto empíricas como metafísicas que la formalista pero ésta tenía a su vez menos dificultades ontoepistémicas que la línea transmutacionista en el sentido de que el o los posibles mecanismos que llevaran a cabo un proceso evolucionista (von Buch, Lamarck, Chambers) eran sugerentes pero no mínimamente convincentes de modo que una mayoría se viera arrastrada a la creencia en el evolucionismo con fundamentación tanto empírica como metafísica (suponiendo, como es mucho suponer, que ambas dimensiones sean separables). El paso que da Darwin en 1858-9 como se ha dicho antes hace que una metafísica subyacente salga a la luz y que la línea formalista se decante en la evolucionista pero sólo en el aspecto propiamente metafísico, porque la empiria en torno a la selección natural, como manifestaba John Stuart Mill, era harto sugerente pero no tenía fundamento empírico relevante alguno.

De hecho, Darwin antes de leer a Malthus seguía en sus especulaciones muy de cerca las ideas de Carpenter que exhortaba a los naturalistas que en vez de preocuparse en descubrir finalidades (adaptaciones, funciones) en el mundo orgánico intentarán descubrir leyes generales como primera providencia, luego ya se podrían concentrar en los "para qués». Es más, Carpenter pensaba que de esa manera la biología y la física estarían a la par a nivel explicativo y la concepción de lo natural (física y biología) se remitiría a una simplicidad epistémica que ontológicamente tenía mucho más sentido. En esta dirección Darwin ponía al mismo nivel ontoepistémico la ley de la gravedad newtoniana (algo que ocurre pero no se puede explicar en la línea de hypotheses non fingo) y una ley transmutacionista, la de la selección natural, que del mismo modo se percibía a la par con la ley de la gravedad aunque no se pudiera explicar.

Como se ha dicho antes de pasada, las tres líneas, la funcionalista, la formalista y la transmutacionista tenían en un principio un común denominador y 
éste era la creencia de lo que se percibía como una naturaleza «existencialmente» perfecta ${ }^{21}$ y en total armonía gracias a las leyes naturales que daban forma y sentido al universo en todas sus manifestaciones. La perfección, claro está, no era ilimitada sino que estaba constreñida por las leyes naturales de manera que era lo más perfecta que podía ser dentro de la cobertura legal existente, o sea que mientras más leyes más limitaciones por lo que en este sentido la perfección posible alcanzada era mayor (por existir más grados de libertad, o sea menos leyes naturales) en la interpretación funcionalista, menor en la formalista y todavía menor en la transmutacionista. De hecho, la nueva ley que descubriera Darwin en el contexto malthusiano quedó como ley de Malthus porque fue este autor el que atrajo la atención de Darwin hacia dicha ley de la que Darwin no había sido totalmente consciente (lo mismo que le ocurriera a Wallace 20 años después).

La idea maestra de Darwin antes de leer a Malthus se centraba en la concepción de una ley de adaptación que se diferenciaba de la que concebían sus coetáneos en un sentido parecido a la especulación clave que Chambers propondría en 1844. Es decir Darwin pensaba, como la generalidad de sus colegas, que cuando algo cambia en las "condiciones de existencia», se genera un cambio correspondiente en los organismos que garantiza su adaptabilidad a las nuevas condiciones de acuerdo a una ley de adaptación global. Pero para la mayor parte de sus colegas ${ }^{22}$ ese cambio orgánico sólo se puede producir dentro de unos límites que son los que definen las especies. Darwin pensaba, de un modo totalmente gratuito por cierto, que en la práctica no había límites o sea que en realidad las especies no existían porque si no su teoría de la selección natural se iría al traste, y otras teorías de tipo saltacionista se le antojaban un tanto inverosímiles aunque no a su amigo y máximo especialista en anatomía comparada Thomas Henry Huxley. De igual modo, como ya se ha indicado, Chambers propu-

${ }^{21}$ El concepto de perfección tenía una connotación ética subyacente de una intensidad colosal porque el problema del «mal» tipificado en el sufrimiento humano y animal estaba siempre en un primer plano e impugnaba emocionalmente de lleno cualquier noción de perfección (sobre todo en el contexto más propiamente inglés).

22 Un disidente notable es el profesor escocés, titular entonces de zoología comparada de la Universidad de Londres, Robert Edmond Grant, lamarckista convencido que interaccionó positivamente con Darwin cuando éste fue a Edimburgo a estudiar medicina aunque por aquel entonces las ideas evolucionistas de Grant no hicieron impacto alguno en el pensamiento del entonces creacionista Darwin a pesar de que éste incluso conociera las ideas evolucionistas de su abuelo paterno Erasmus. 
so en su momento, tan gratuitamente como Darwin lo hizo en el suyo, que la prolongación de la gestación embriológica es la que daba lugar a una nueva especie lo que traducía el idealismo de los Naturphilosophen en algo palpable. Wallace, cuatro lustros después, pensó como Darwin con el mismo nivel de gratuidad epistémica. Incidentalmente Darwin también deducía que la nueva adaptación compensatoria se fijaba en el embrión. En efecto, la conexión Chambers-Darwin-Wallace era bastante más íntima que en lo que en general se da que pensar.

Darwin en sus cuadernos de notas también glosa sobre las que considera como cuatro leyes de la herencia orgánica en los organismos, a saber, ley 1: la herencia orgánica normal es por «mezcla de sangres», ley 2: la ley de los híbridos con las particularidades de la producción de esterilidad o de reversión de caracteres, ley 3: los caracteres que se fijan por su larga permanencia «en la sangre» o ley de Yarrell y ley 4: los problemas que produce la consanguinidad y que hace que los cruces correspondientes se asemejen a la reproducción asexual. Que duda cabe que estas leyes eran asimismo del dominio público sobre todo entre agricultores y ganaderos que es de donde las entresaca Darwin. La herencia por mezcla de sangres facilitaría la uniformidad (unidad temporal) de la especie así como la adaptación progresiva a los cambios de medio persistentes pero no así a los ocasionales (igualmente conocimiento generalizado). Un experto en problemas de herencia y generación en la época (sobre todo en conexión con la tercera ley), además de ictiólogo y ornitólogo notable, es William Yarrell a quien Darwin estudia profusamente y desde luego en sus cuadernos de notas es el autor más citado después de su padre y su mentor científico Charles Lyell (el cuarto más citado es el ornitólogo John Gould cuya decisión de clasificar como especies lo que Darwin pensaba que eran variedades es lo que le provoca la «crisis» evolucionista en nuestro protagonista).

De manera que Darwin en una primera etapa sobre todo estudia la ciencia natural de su tiempo aunque el patronazgo inicial de Charles Lyell hace que sobresalga casi de inmediato en especulaciones propiamente geológicas. Seguidamente, la experiencia que tuvo en su día en Edimburgo con Grant le reconduce asimismo con enorme éxito al estudio de los percebes tanto fósiles como vivientes. Finalmente, su mejor amigo, el botánico notable Joseph Dalton Hooker, director del botánico de Londres (Kew Gardens), está ahí arropándole científicamente en su famoso estudio sobre las orquídeas y otros estudios botánicos posteriores. Y como una segunda actividad extra-oficial Darwin intenta teorizar sobre el origen de las 
especies, y lo que si consigue gracias especialmente a sus interlocutores alemanes (especialmente Ernst Haeckel primero y August Weismann después) es potenciar un impulso naturalizador de la condición humana que explosiona existencialmente muchos años después (de hecho ya en nuestro tiempo) dándole fuerza explicativa al nihilismo existencial que caracteriza nuestra tesitura presente.

\section{El equívoco «efecto Malthus»}

La relectura de la obra de Malthus Essay on the Principle of Population (1798, $1^{\text {a }}$ ed.) un 28 de septiembre de 1838 por parte de Darwin que según sus propias palabras le proporciona una teoría con la que puede trabajar - que es la supuesta teoría de la selección natural - lo único de lo que realmente le hace consciente es que parte importante del medio de un ser vivo son los otros seres vivos de manera que el desafío de un ser vivo que compite por los mismos recursos, es decir que pertenece no ya a la misma especie sino a la misma población supone, como en el caso del medio inorgánico, la inducción de variaciones compensatorias. Pero claro este tipo de variación es en efecto una variación que compite con otra enfrentada de manera que la variación que mejor se exprese es la que anula a la otra que no puede superarla, de modo que ocurre como en la extinción orgánica, es decir, hay extinción orgánica cuando la variación del medio no se puede compensar con la variación orgánica porque ésta se presenta como una macrovariación imbatible a la manera en efecto de una catástrofe local cómo dice Darwin basándose en Lyell. Es decir que en principio, y de hecho así ocurre en los siguientes tres meses después de leer a Malthus, las variaciones no son estrictamente accidentales sino que simplemente no pueden contrarrestar las variaciones que se presentan en la otra parte que es la del organismo que compite (como compite el medio que en este sentido es como si fuera otro organismo y así de hecho lo manifiesta Darwin en su Origin).

O sea que desde esta perspectiva la teoría de la selección natural (del organismo con respecto al medio) ha existido siempre de un modo tácito y todos los coetáneos de Darwin la han asumido sin más problema. Lo que quiere creer Darwin es lo que ha intentado demostrar fallidamente desde un primer momento y es exponer que esa acción de alguna manera rompa los límites de la especie y sea de aplicación indefinida y, claro ésta, es la crítica general que Darwin no asume, aparte de otras críticas discutiblemente menores que recibe de sus coetáneos (Him- 
melfarb, 1959, Vorzimmer, 1972, Hull, 1973). O sea que los límites de la especie, o el plateau desde la selección artificial (como se dice ahora), lo que induce a pensar es precisamente que esos límites están ahí y Darwin no dice como se soslayan. Es verdad que el naturalista inglés en algún momento ( $5^{\mathrm{a}}$ edición del Origin) manifiesta que de vez en cuando, raramente, con el tiempo esos límites se rompen de un modo análogo a como los paleontólogos actuales Steven Jay Gould $(R I P)$ y Niles Eldredge manifiestan que la evolución tal y como la concebimos ocurre en períodos en que hay mucha actividad mutacional y que los otros períodos entre medias son a la sazón estáticos (teoría del equilibrio pautado/puntuado).

A principios de marzo de 1839 en el cuadernito de notas E Darwin ya habla de variaciones aleatorias es decir de variaciones que nada tienen que ver con las supuestas necesidades adaptativas de los organismos, pero de nuevo ese cambio de chip es puramente gratuito en el sentido que Darwin manifiesta a menudo (contestando también numerosas críticas en este sentido) que el origen de las variaciones es desconocido, de modo que aplicando en cierto modo el conocimiento actual que se empareja con el de Darwin antes de concebir las variaciones como aleatorias lo que tenemos es un proceso de homeóstasis generalizado. Es más, Darwin, como la mayoría de sus coetáneos, y muy especialmente Wallace, piensa que las variaciones que seleccionan los mejoradores y criadores en general no son más que variaciones patológicas que hacen que la aparición de esos organismos artificiales (misfits) o bien una vez de nuevo en libertad reviertan al estado natural o bien simplemente ya no puedan sobrevivir sin el apoyo del hombre. Claro, hablar de variaciones accidentales/indiferentes/aleatorias resuelve de golpe tres problemas: el de la selección natural, el de la selección artificial y el de la teleología como último remanente de la historia natural teologizada. Sin embargo, esta última hipótesis de Darwin una vez más es gratuita, aunque desde una perspectiva naturalista extrema sea la más aceptable y es algo que no ha calado en la teoría de la evolución vigente hasta casi el primer centenario del Origin en 1959. Es más, en la teoría alternativa a la selección natural denominada direccionalismo (siendo uno de sus principales defensores Stuart Kauffman), la aleatoriedad de las mutaciones tiene un papel menor como incidentalmente también lo tenía para Darwin en el sentido de que éste nunca renuncia a la existencia de un "plan natural» donde los detalles sin importancia se dejan a cargo de las mutaciones aleatorias. Ciertamente, a la luz de estas consideraciones, el Darwin que se proyecta desde la ortodoxia darwiniana de nuestro tiempo no tiene mucho que ver con el Darwin real. 


\section{El problema de los problemas}

Si el «misterio de los misterios» era el origen de las especies (la expresión es de John Herschel utilizada en su recensión de los Principles of Geology de Lyell), misterio que se atenúa por el trasiego dialéctico en que incurren primero Chambers y luego Darwin y Wallace. Y es que si bien éstos no resuelven el problema por lo menos alimentan la creencia en una teoría evolucionista a la que luego los anglo-americanos Cope, Hyatt, Osborne y Marsch, entre bastante otros, le darían un colorido ortogenético lamarckiano y, acto seguido, al siglo siguiente fundamentalmente con las ideas del holandés Hugo de Vries, así como del clérigo agustino Johann Mendel puesto al día (especialmente por Thomas Hunt Morgan y su equipo) las ideas de Darwin vuelven a resurgir debidamente matematizadas (Fisher, Haldane y Wright) y reinterpretadas desde la historia natural sobre una base muy amplia (Theodosius Dobzhansky, George Gaylord Simpson, Ernst Mayr, Julian Huxley, George Ledyard Stebbins). Por todo ello se llega a una ortodoxia darwiniana que a pesar de disensiones notables (Motoo Kimura, Steve Kauffman) sigue marcando la pauta teórica en la actualidad.

Pero la tesitura se complica con el «problema de los problemas» (o la "cuestión de las cuestiones» como diría T. H. Huxley). En la mente de Darwin está claro que el hombre no es un ser vivo cualitativamente distinto de cualquier otro, aunque ocurre como con la selección natural sólo que peor, es decir, ¿cómo se demuestra? Sus amigos naturalistas más cercanos publican teorías al respecto por las cuales el hombre es especial (por ejemplo, Lyell y sus The Geological Evidences of the Antiquity of Man, 1863) o no lo es (por ejemplo Thomas H. Huxley's Evidence as to Man's Place in Nature, 1863, o John Lubbock's Pre-historic Times, 1865). Es más, Lyell le convence a Wallace de sus ideas acerca de la naturaleza «espiritual» del hombre ${ }^{23}$, ideas que Wallace amplia con pensamientos de su propia cosecha y Darwin se queda solo ante "el peligro» de la trascendencia, por lo que decide mojarse y escribir The Descent of Man (1871, 1. ${ }^{\mathrm{a}}$ ed.) que es una obra desproporcionada en su temática, desigual en su desarrollo y mucho peor argumentada que su «origen de las especies». Además el crítico más incisivo que Darwin tuviera nunca, el citado Mivart, refleja lo que estima como vacuidad lógicoempírica en los argumentos de Darwin en el Quaterly Review amén de en su

23 Para la temática en torno al origen del hombre en tiempo de Darwin véase Schwartz (1984). 
Genesis of Species (1871) hasta el punto que en la sexta y última edición del Origin (1872) Darwin tiene que añadir un capítulo para tratar de rebatir a tan colosal crítico.

A la altura del Descent of Man Darwin ya ha perdido gran parte de su fe si no en la teoría de la selección natural sí en su capacidad de convencer a otros y las concesiones a sus críticos llegan a límites insospechados. Para empezar, Darwin piensa que en el origen del hombre la selección natural ha tenido un papel mínimo, mientras que el papel principal se debe a la selección sexual, a los efectos lamarckianos del uso y la falta de uso (besoin intérieur), a la acción directa del medio, a los efectos secundarios por correlación de efectos así como a causas que ignoramos. Darwin en efecto está perplejo en el sentido de que lo que antes pensaba que era una especie de panseleccionismo ahora se convierte en la consideración de que la mayor parte de las características orgánicas son neutras, es decir, ni útiles ni inútiles. Véase el siguiente extracto del Descent (que fue auténtica dinamita en manos de los críticos de Darwin, especialmente de Mivart):

Una parte muy importante aunque indefinida se puede atribuir sin lugar a dudas a los resultados directos e indirectos de la selección natural; pero ahora reconozco... que en las ediciones anteriores de mi «Origen de las Especies» probablemente atribuí demasiado a la acción de la selección natural o supervivencia del más apto ... Antes no había considerado lo suficientemente la existencia de muchas estructuras que se presentan, en la medida que se pueda apreciar, como si no fueran ni beneficiosas ni injuriosas y creo que esto es uno de los detalles más importantes que se me han pasado por alto. A manera de excusa, al menos en cierta medida, puedo alegar que tenía dos objetivos en perspectiva, primeramente que las especies no han sido creadas por separado y, en segundo lugar, que la selección natural había sido el agente principal de cambio, aunque apoyada en gran medida por los efectos heredados del hábito, y ligeramente por la acción directa de las condiciones circundantes. Sin embargo no me era posible anular la influencia de mis creencias previas, ampliamente prevalentes por aquel entonces, de que cada especie había sido creada intencionalmente y esto me condujo a asumir tácitamente que cada detalle estructural, exceptuando los órganos abortados, tenía un cometido aunque no se percibiera una función específica. Quien quiera que piense así le daría a la acción de la selección natural demasiada fuerza tanto en el pasado como en la actualidad ... Si me he equivocado en haberle atri- 
buido a la selección natural una gran fuerza, algo que estoy lejos de admitir, o de haber exagerado su poder lo que es probable en sí mismo, por lo menos espero haber tenido éxito en mi contribución a refutar el dogma de las creaciones especiales $^{24}$

Dicho párrafo lo toma Mivart para minar todavía más el trabajo teórico de Darwin y éste recoge velas porque en la segunda y última edición del Descent (1874) Darwin se declara convencido de que «las numerosisimas estructuras que se nos muestran como sin utilidad alguna, se probarán que son vitales y que por tanto estarán cubiertas por la selección naturali ${ }^{25}$. Además, Darwin admitía en esa primera edición que "extrañas y muy señaladas peculiaridades estructurales» ${ }^{26}$ aparecían sin causas conocidas y se perpetuaban sin remitirse a ningún tipo de selección o adaptación. Claro, este tipo de admisión dejaba el camino libre para esa consideración clásica sobre que esas estructuras eran macrovariaciones naturales - y quizá de origen divino - por las que al fin y a la postre se explicaba el origen de las especies y muy especialmente el origen del hombre, de manera que en la segunda edición esas «muy señaladas peculiaridades» se convertían en algo «sin importancia fisiológica» ${ }^{27}$.

Los argumentos arriesgados del Descent así como las vueltas atrás debido sobre todo a las críticas contundentes de Mivart hacen que los argumentos de la obra de Darwin sobre el hombre dejen bastante que desear en comparación a los razonamientos del Origin y es que, claro, explicar el origen del hombre exige matizaciones mucho más cuidadosas que hacer lo propio con, por ejemplo, el origen de las palomas (otro tema puntual favorito de Darwin) aunque creamos que esencialmente estamos hablando de lo mismo.

En efecto, una cosa es «creer» y otra «demostrar» como manifestaba el físico William Hopkins en su crítica del Origin $^{28}$, aunque entre medias haya una tierra de nadie todo lo amplia que se quiera. El naturalismo extremo que hizo presa en Darwin así como en una reducida minoría de sus coetáneos (Huxley, Tyn-

${ }^{24}$ Descent of Man, 1. a ed., parte I, págs. 152-3, John Murray, Londres.

25 Descent, 2. ${ }^{\mathrm{a}}$ ed., 1874, p. 92, John Murray, Londres.

26 Descent, $1 .^{\mathrm{a}}$ ed., parte II, p. 387.

27 Descent, 2. ${ }^{\mathrm{a}}$ ed., 1874 , p. 93.

${ }^{28}$ Hull (op. cit). 
dall) hoy día ${ }^{29}$ es por el contrario moneda de curso legal entre la comunidad científica. Pero esto último sucede no porque al contrario de en época de Darwin se haya demostrado algo que Darwin no pudiera dilucidar entonces, sino porque simple y llanamente hoy contemplamos la naturaleza general y la propia no a través de los ojos de Darwin — como se suele estipular por admiradores más que seguidores- no, ni mucho menos, sino que contemplamos con los mismos ojos que lo hacía Darwin lo que aunque lo parezca no es igual. Darwin no es ni un profeta ni un visionario ni siquiera un adelantado de su tiempo, toda consideración de esa índole sería historia presentista (Whiggish). Sino que el naturalista inglés es alguien que junto a algunos otros se convenció en su medio particular por razones metafísicas, que a él le parecieron propiamente científicas, que la realidad que vivimos es inhóspita e indiferente hacia nuestros intereses individuales cualesquiera que éstos sean ${ }^{30}$.

\section{Bibliografía (fuentes secundarias)}

BAISDELL, M. (1982). «Natural ethology and Nature`s disguises». Journal of the History of Biology, 15:163-89.

BECK, N. (2009). «The Origin and political thought: From liberalism to Marxism» en Ruse and RICHARDS, eds., pp. 295-316.

Brown, F. B. (1986). «The evolution of Darwin's theism». Journal of the History of Bio$\log y, 19: 1-45$.

BurkhardT, F. (1974). England and Scotland: The Learned Societies en The Comparative Reception of Darwinism (ed. T. F. Glick). University of Texas Press, Austin y Londres, pp. 32-74.

Bynum, W. F. (1984). Charles Lyell's Antiquity of Man and its critics. Journal of the History of Biology, 17:153-87.

Castrodeza, C. (1988). Teoría Histórica de la Selección Natural. Alhambra Madrid).

${ }^{29}$ Hoy día en efecto la que podemos llamar línea agnóstica Huxley-Tyndall equivaldría a la línea ateológica Dawkins-Atkins.

30 Para una serie reciente de estudios notables en torno a las connotaciones filosóficas de Darwin y el darwinismo se puede consultar Hösle y otro, eds. (2005) aunque ninguno de los autores implicados en esa obra se centran en la temática desde la perspectiva amplia histórico-metafísica de este artículo. 
- (2009). La Darwinización del Mundo. Herder, Barcelona.

Cornell, J. F. (1987). God's magnificent law: The Bad influence of theistic metaphysics on Darwin's estimation of natural selection. Journal of the History of Biology, 20:381-412.

CoRsi, P. (2005). Before Darwin: Transformist Concepts in European Natural History. Journal of the History of Biology, 38:67-83.

Crowther, J. G. (1982). Founders of British science : John Wilkins, Robert Boyle, John Ray, Christopher Wren, Robert Hooke, Isaac Newton. Greenwood Press, West port, Connecticut.

Desmond, A. (1984). «Robert E. Grant: The Social Predicament of a Pre-Darwinian Transmutationist». Journal of the History of Biology, 17:189-223.

Gould, S. J. (1977). Ontogeny and Phylogeny. The Belknap Press of Harvard University Press, Cambridge, Mass.

FranCIS, M. (2007). Herbert Spencer and the Invention of Modern Life. Cornell University Press, Ithaca.

Gillespie, N. C. (1979). Charles Darwin and the Problem of Creation. University of Chicago Press, Chicago y Londres.

Himmelfarb, G. (1959). Darwin and the Darwinian Revolution. Norton, Nueva York.

Hösle, V. y ILlies, C. eds. (2005). Darwinism and Philosophy. University of Notre Dame Press, Indiana.

Hull, D. (1973). Darwin and his Critics. Harvard University Press, Cambridge, Mass.

Laublicher, M. D. y Maienschein, J. (2008). Form and function in developmental evolution. Cambridge University Press. Cambridge (R:U) y Nueva York.

MARSHALL, J. (2006). John Locke, toleration, and early Enlightenment culture : religious intolerance and arguments for religious toleration in early modern and "early Enlightenment» Europe. Cambridge University Press. Cambridge (R:U) y Nueva York.

Ospovat, D. (1981). The Development of Darwin's Theory: Natural History, Natural Theology \& Natural Selection 1838-1859. Cambridge University Press, Cambridge (R.U.).

Ruse, M. y Richards, J. R. eds. (2009). The Cambridge Companion to the "Origin of Species». Cambridge University Press, Cambridge (R.U.).

Russell, E. S. (1916). Form and Function: A contribution to the history of animal morphology. John Murray, Londres. 
SALAdin, K. S. (2009). Anatomy o physiology : the unity of form and function. McGrawHill, Dubuque.

SChwartz, J. S. (1984). Darwin, Wallace, and the Descent of Man. Journal of the History of Biology, 17: 271-89.

SECORD, J. A, (1991). Edinburgh Lamarckians:Robert Jameson and Robert E. Grant. Journal of the History of Biology, 24:1-18.

SECORD, J. A, ed. (1994). Vestiges of the natural history of creation and other evolutionary writings de Robert Chambers ; edición y nueva introducción de James A. Secord. University of Chicago Press, Chicago.

STEnhouse, J. (1990). Darwin's captain: F. W. Hutton and the Nineteenth-Century Darwinian debates. Journal of the History of Biology, 23:411-42.

Vorzimmer, P. J. (1972). Charles Darwin: The years of controversy (the Origin of Species and its critics). University of London Press Ltd, Londres.

Recibido: 3/09/2009

Revisado: 10/09/2009 
